UCRL-JC-129813

PREPRINT

\title{
Diagnosing Hot Electron Production by Short Pulse, High Intensity Lasers Using Photonuclear Reactions
}

T. W. Phillips, M. D. Cable, T. E. Cowan, S. P. Hatchett, E. A. Henry, M. H. Key, M. D. Perry, T. C. Sangster, M. A. Stoyer

This paper was prepared for submittal to the 12th Topical Conference on High-Temperature Plasma Diagnostics Princeton, NJ

June 7-11, 1998

June 2, 1998

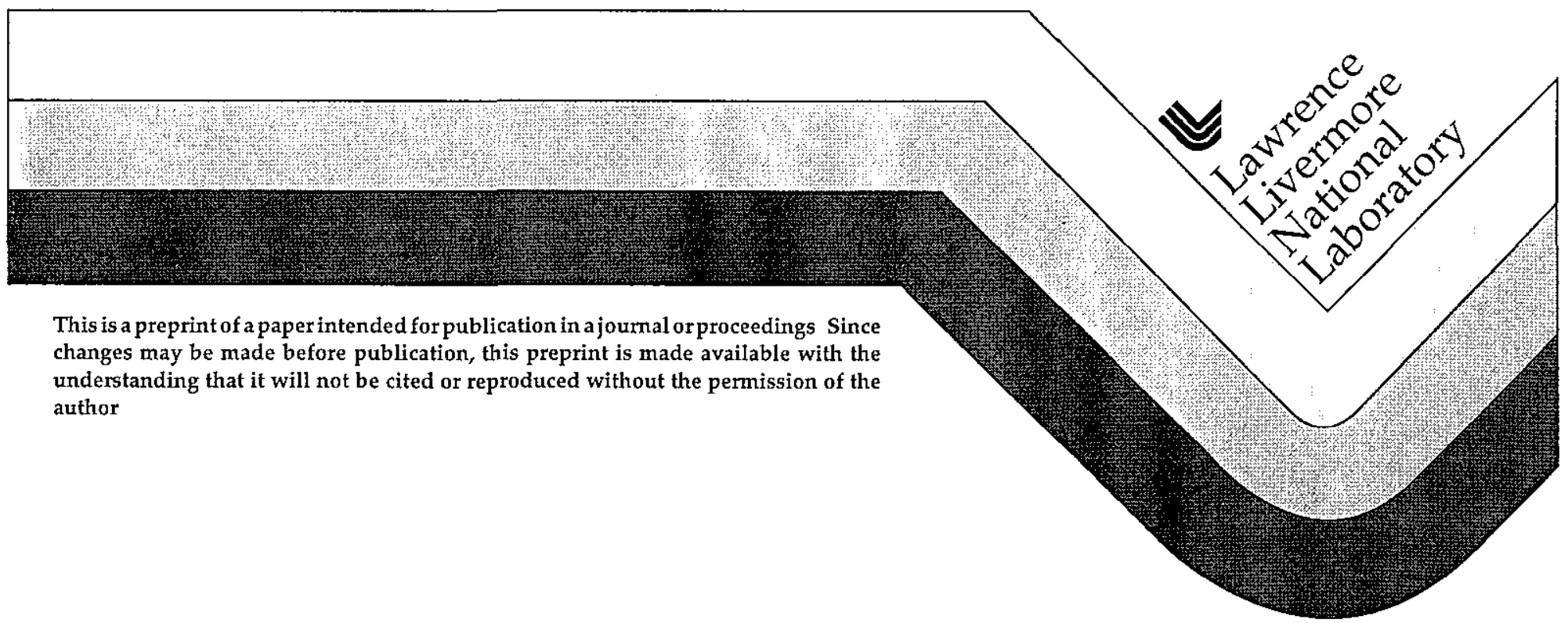




\section{DISCLAIMER}

This document was prepared as an account of work sponsored by an agency of the United States Government Neither the United States Government nor the University of California nor any of their employees, makes any warranty, express or implied, or assumes any legal liability or responsibility for the accuracy, completeness, or usefulness of any information, apparatus, product, or process disclosed, or represents that its use would not infringe privately owned rights Reference herein to any specific commercial product, process, or service by trade name, trademark, manufacturer, or otherwise, does not necessarily constitute or imply its endorsement, recommendation, or favoring by the United States Government or the University of California The views and opinions of authors expressed herein do not necessarily state or reflect those of the United States Government or the University of California, and shall not be used for advertising or product endorsement purposes 


\section{Diagnosing hot electron production by short pulse, high intensity lasers using photonuclear reactions.}

T. W. PHILlipS, M. D. CABLE, T. E. COWAN, S. P. HATCHETT, E. A. HENRY, M. H. KEY, M. D. PERRY, T. C. SANGSTER, AND M. A. STOYER

Lawrence Livermore National Laboratory., Livermore, California, 94550

Solid targets irradiated with $10^{19}$ watts $/ \mathrm{cm}^{2}$ or greater of 1 micron light in picosecond pulses are found to be radioactive. The strongest activities observed are the result of photonuclear reactions in which an energetic photon excites the nucleus sufficiently to produce particle emission leaving a radioactive daughter. The photo reaction cross sections are known for a wide range of nuclei and provide a quantitative measure of the photon flux produced in the target. Both the delayed daughter activities and measurements of the prompt particles emitted in the reaction can be used as diagnostics. Examples of these techniques applied in diagnosing experiments at the Nova laser facility adapted to generate petawatt pulses using chirped pulse amplification will be presented. These results will be compared with bremsstrahlung photon spectra calculated using electron spectra measured in a magnetic spectrograph.

Diagnostic techniques are being developed to characterize hot electrons produced by intense short pulse laser beams interacting with various targets. The LLNL Petawatt laser [1] (PW) is presently the world's highest power Chirped Pulse Amplification system, and as such provides a new tool for studying ultra-relativistic laserplasma processes including ultra-high gradient acceleration of electrons. Applications of this source of energetic electrons include fast ignition of compressed ICF plasmas[2], flash radiography[3], and laser particle accelerators.[4] The Petawatt laser, at the Lawrence Livermore National Laboratory NOVA facility, uses one arm of the NOVA Nd:glass amplifier chain to amplify a stretched pulse from the Ti:sapphire oscillator to $\sim 500 \mathrm{~J}$. The beam is quite large $(46 \mathrm{~cm}$ diameter) to avoid damage to the subsequent reflective optics, and requires state-of-the-art $75 \mathrm{~cm}$ diameter gratings to recompress the beam to $\sim 450 \mathrm{fs}$. The final focus optic is an $80 \mathrm{~cm}$ diameter on- 
axis parabola, with which a $\sim 20 \mu \mathrm{m}$ diameter focal spot has been achieved.

Focused intensities in the range of $10^{21} \mathrm{~W} / \mathrm{cm}^{2}$ produce enormous electromagnetic fields at the laser focus $\left(E @ 10^{14} \mathrm{~V} / \mathrm{m}\right.$, and $B @ 3 \times 10^{5}$ Tesla, at $10^{21} \mathrm{~W} / \mathrm{cm}^{2}$ ) and the motion of the electrons in the target plasma is fully relativistic. Their cycleaveraged oscillation or "quiver" energy, <E>, can exceed $10 \mathrm{MeV}$, where

$$
\text { (1) }\langle E\rangle=m c^{2}\left[1+2 U_{p} / m c^{2}\right]^{1 / 2}
$$

where $U_{p}=9.33 \times 10-14 \mathrm{I}\left(\mathrm{W} / \mathrm{cm}^{2}\right) \lambda^{2}(\mu \mathrm{m})$ is the non-relativistic ponderomotive potential. The resulting distribution of electron energies in the target resembles a Maxwellian,[3]

(2) $N(E) d E \propto(E /<E>)^{1 / 2} \exp (-E /<E>) d E$

with mean energy given by Eq. 1, which extends beyond the threshold for nuclear activation. Characterization of the laser-target interactions requires the measurement of the energy spectrum of electrons and photons within the target and escaping from it. A number of diagnostic techniques have been developed to obtain this information. In this paper we describe techniques using electro- or photonulcear processes to diagnose the high-energy electron emission from laser-solid interactions and compare them with other techniques and calculation.

Electromagnetic excitation of nuclei proceeds along two paths, direct electroexcitation and photoexcitation. Electroexcitation is reduced relative to photoexcitaion by the fine structure constant, $1 / 137$, but photoexcitation requires the conversion of electron energy to photons by the bremsstrahlung process which is proportional to the path length in the material. The cross section for this process is proportional to the square of the atomic number of the material. A complication in the electroexcitation case is that the scattered electron in the final state can carry away energy. Therefore, the nucleus can be excited to any level up to the electron's total energy. For thin low $Z$ targets electroexcitation can be the dominant photoexcitation.

The nuclear response to electromagnetic excitation depends on the energy and the nuclear mass of the target atom. At the lowest energies isomeric states of the nucleus can be excited whose lifetimes may permit detection by observing the gamma decay of the excited state. At higher energies particle emission can be observed first to individual states and then to an overlapping continuum of states. This particle emission cross section typically peaks at the 
giant resonance energy between 10 and $20 \mathrm{MeV}$.[5] For most nuclei the spectrum of prompt particles for a monoenergetic excitation is sufficiently complex that uniquely interpreting the spectrum of excitation from the emission spectrum is not possible. The exception is deuterium for which the emitted neutron and proton energies are proportional to the excitation energy. This and the known excitation cross section permit the extraction of the incident spectrum. For heavy nuclei $(A>40)$ particle emission even at a single energy of excitation is a complex continuum that may be divided into direct, preequilibrium, and equilibrium regimes.[6] Direct and preequilibium mechanisms produce the high energy tail of the particle distribution with some asymmetry in angular distribution. The equilibrium component is an isotropic maxwellian distribution with a temperature of $\sim 1 \mathrm{MeV}$.[7] Finally activation measurements can be used to derive the excitation flux over an energy interval corresponding to the width of the giant resonance. By appropriate selection of targets with varying thresholds and giant resonance energies the photon spectrum can be mapped out.

To maximize the bremsstrahlung production from the hot electrons and measure its spectrum, a $A u$ target backed with $\mathrm{CD}_{2}$ was designed. LaNSA[8] is used to measure the photoneutron emission spectra from the target by the time-of-flight technique. LaNSA is an array of 960 scintillation detectors at $21.6 \mathrm{~m}$ from PW target giving it excellent energy resolution. The detector is at an angle of $69^{\circ}$ or $111^{\circ}$ depending on the direction of the laser beam (Fig 1). For each detector the arrival time (when the signal exceeds a certain threshold) and the length of time it remains above the threshold are digitized and stored. Multiple threshold crossings can be recorded for each detector. The neutron detection efficiency is estimated from the intrinsic LaNSA efficiency, the flight path transmission and solid angle. For neutrons at $2.45 \mathrm{MeV}$ this calculation gives $2.05 \times 10^{-7}$ counts/channel/source neutron. A problem for LaNSA in this application is its sensitivity to the prompt $\gamma$-flash from the target which can paralyze it during the neutron arrival time.

Analysis of the photoneutron spectra from radiography targets uses a Monte Carlo simulation (ITS[9]) to model the electron/photon transport in the target. Electron spectra escaping from the PW target, measured with a compact, permanent magnet electron spectrometer using nuclear emulsion track detectors[10], constrain this calculation. An iterative procedure achieves reasonable agreement between the measured and calculated electron flux as 
shown in Fig. 2 The calculated photon fluxes in the $\mathrm{Au}, \mathrm{Cu}$, and $\mathrm{CD}_{2}$ regions are weighted by their respective photonuclear cross sections to give the expected neutron yield.

\begin{tabular}{ccc} 
Sources & $\begin{array}{c}\text { (Gamma, N) } \\
\text { Yield }\end{array}$ & $\begin{array}{c}\text { Neutrons } \\
>1 \mathrm{MeV}\end{array}$ \\
\cline { 2 - 3 } $\begin{array}{c}\text { Target } \\
\text { CD2 }\end{array}$ & $\begin{array}{c}7.51 \times 10^{6} \\
\text { Au }\end{array}$ & $2.44 \times 10^{6}$ \\
$\begin{array}{c}\text { Cu } \\
\text { Chamber } \\
\text { Al }\end{array}$ & $6.10 \times 10^{6}$ & $6.00 \times 10^{5}$ \\
Total & $9.70 \times 10^{5}$ & $1.00 \times 10^{5}$ \\
\cline { 2 - 3 } & $5.90 \times 10^{5}$ & $3.00 \times 10^{5}$ \\
\hline
\end{tabular}

The measured LaNSA yield for $\left.E_{n}>1 \mathrm{MeV}\right), 3.45 \times 10^{6}$ and the calculated yield $\left(E_{n}>1\right), 3.44 \times 10^{6}$ are in surprisingly good agreement. The spectral shapes(fig 3 ) also match well giving added confidence in our knowledge of the electron spectra above $4 \mathrm{MeV}$.

The activation of $\mathrm{PW}$ targets also demonstrates the existence of photonuclear processes with $\mathrm{E}_{\gamma}>10 \mathrm{MeV}$ in the laser-target interaction. Targets with $\mathrm{Au}$ and $\mathrm{Cu}$ and $\mathrm{Au}, \mathrm{Ni}, \mathrm{In}$ and $\mathrm{Ti}$ layers have been studied. The target gamma ray activity is measured[11] to identify and quantify the reactions induced by the PW laser beam. $\mathrm{Cu}^{63}(\gamma, n)$ at a yield of $4.50 \times 10^{7}$ reactions and $\mathrm{Ni} 58(\gamma, n)$ yield 9.08 $x 10^{5}$ reactions are observed in $\mathrm{Cu}$ and $\mathrm{Ni}$ target foils as well as Au197 $(y, n)$. The photon flux required to reproduce the activity observed in $\mathrm{Cu}$ and $\mathrm{Ni}$ components of the radiography and activation targets is determined from the masses and geometry using the measured photonuclear cross sections and assuming the photon source is a point at the gold surface. The resultant flux is plotted at the average energy determined by weighting the cross section with the exponential spectral shape of the flux. These results are compared with other measurements and calculation in fig 4.

Secondary activation by photoneutrons has also been observed in the $\mathrm{Au}, \mathrm{Ni}$, In and $\mathrm{Ti}$ targets. This activation provides an additional check on the flux of photoneutrons produced by photons with energies above the photonuclear threshold in the targets. The relative production of neutron activation in the target materials 
was estimated using a neutron transport calculation (TART[12]). The neutron source strength and spectrum in each zone is determined using the measured photonuclear cross sections and neutron spectra for its materials and the photon flux from an ITS calculation based on the measured electron spectra as previously described.

\section{Table of Neutron Activation Calculation versus Measurement}

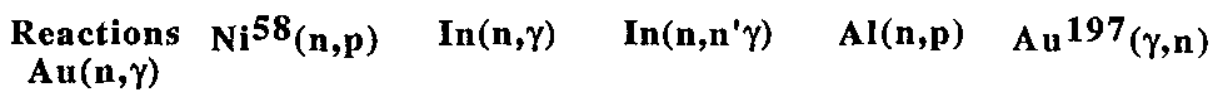

\begin{tabular}{|c|c|c|c|c|c|c|}
\hline $\begin{array}{c}\text { Calculated } \\
\text { Activity }\end{array}$ & $2.53 \times 10^{3}$ & $4.21 \times 10^{3}$ & $1.16 \times 10^{4}$ & $3.77 \times 10^{4}$ & $3.09 \times 10^{1}$ & $4.67 \times 10^{6}$ \\
\hline $\begin{array}{l}\text { Measured } \\
\text { Activity }\end{array}$ & $8.12 \times 10^{4}$ & $\begin{array}{c}\text { not } \\
\text { detected }\end{array}$ & $1.58 \times 10^{4}$ & $3.17 \times 10^{5}$ & $\begin{array}{c}\text { not } \\
\text { detected }\end{array}$ & $3.97 \times 10^{6}$ \\
\hline half-life & $3.88 \times 10^{3}$ & $1.02 \times 10^{5}$ & $5.44 \times 10^{1}$ & & 9.46 & $8.90 \times 10^{3}$ \\
\hline $\begin{array}{l}\text { dpm calc. } \\
\text { prompt }\end{array}$ & $4.52 \times 10^{-1}$ & $2.86 \times 10^{-2}$ & $1.48 \times 10^{2}$ & & 2.27 & $3.63 \times 10^{2}$ \\
\hline
\end{tabular}

Calculation and measurement agree for $\ln (n, \gamma)$ but not for $A u(n, \gamma)$. Au may be a problem due to its low decay rate and background interference. The ratio of $\mathrm{Au}$ activity due to $(\gamma, n)$ versus $(n, \gamma)$ is noted to vary from target to target. Further simulations of these targets may uncover the cause of this discrepancy. The discrepancy for $\ln \left(n, n^{\prime}, g\right)$ is due to contributions from photon and electron inelastic scattering. The $A u(g, n)$ difference is attributed to the loss of activated Au due to vaporization by the laser beam.

Finally, targets to study buried layer heating consisting of a thin $10 \mu \mathrm{m} \mathrm{CH}$ foil, a $1 \mu \mathrm{m} \mathrm{Al}$ foil and $500 \mu \mathrm{m} \mathrm{CD} 2$ foil were fielded. These thin low $Z$ targets produce very little bremsstrahlung and the electronuclear process

$$
e^{-}+A \rightarrow A-1+n+e^{-}
$$

dominates the production of neutrons in the $\mathrm{CD}_{2}$. Fits to LaNSA data assuming electrodisintegration of deuterium and two possible shapes for the exponentially falling electron distribution is shown in fig 5. Electron spectra are not available for these targets to experimentally select between these distributions. 
Hot electron production by short pulse, high intensity lasers has been diagnosed. Energetic electrons, prompt photoneutrons, and photonuclear activation have been observed consistent with proposed mechanisms of intense, short-pulse laser- target interactions. Photoreactions provide a quantitative diagnostic tool to study hot electron production which complements measurements of radiation dose and electron spectra. Prompt neutrons from deuterium photoreactions can measure the photon flux at energies important to the applications of this hot electron source.

Photoactivation measures the flux in the high energy tail of the hot electron distribution and further verifies the photoneutron production by neutron activation of target nuclei. Electronuclear excitation is also a source of prompt particles and activation and may be needed to diagnose thin targets of interest for fast ignition. In the future clever target designs will extend these diagnostics to give the angular variation of the electron source. As the PW laser is improved detection techniques for prompt particles must be developed which are not paralyzed by the strong prompt gamma flash.

This work was performed under the auspices of the U.S. Department of Energy by the Lawrence Livermore National Laboratory under Contract No.W-7405-Eng-48.

1. M.D. Perry, B.C. Stuart, D. Pennington, G. Tietbohl, J.A. Britten, C. Brown, S. Herman, J. Miller, H.T. Powell, M. Vergino and V. Yanovsky, "Petawatt Laser Pulses," submitted to Optics Letters.

2. M. Tabak et al., Phys. Plasmas 1, 1626 (1994).

3. S.C. Wilks, W.L. Kruer, M. Tabak and A.B. Langdon, Phys. Rev. Lett. $\underline{69}, 1383$ (1992).

4. T.E. Cowan, S.C. Wilks, P.E. Young, D.M. Pennington, M.D. Perry, X. Shi, K. van Bibber et al., "Proposal for Laser Acceleration Experiments at the LLNL Petawatt".

5. B. L. Berman , At. Data Nucl. Data Tables 15, 319, (1975)

6. M. Blann, B. L. Berman and T. T. Komoto, Phys. Rev. C28,2286,(1983)

7. G.S. Mutchler, "The Angular and Energy Spectra of Photoneutrons from Heavy Elements", MIT-2098-224 (1966), Mass. Inst. of Tech

8. M. B. Nelson and M. D. Cable, Rev. Scl. Instrum. 63, 4874 (1992)

9. J. A. Halbleib and T. A. Mehlhorn, Nucl. Sci. Eng. 92,338 (1986)

10. T. E. Cowan, et. al. "High energy electron emission and laser-assisted nuclear transmutation in petawatt laser-solid interactions"

11. E. A. Henry, M. A. Stoyer Private communication

12. A Edwards, J Kimlinger and E Plechaty, TART Input Manual, UCID-17026, Rev 3.0.3, Feb. 24, 1992, Lawrence Livermore National Laboratory 


\section{Figure Captions}

Figure 1 Schematic view of LaNSA - petawatt experiment

Figure 2 Comparison of ITS input electron spectrum with measured and calculated escaping electron spectrum at $30^{\circ}$

Figure 3. Comparison of LaNSA measured neutron flux with calculation using ITS photon flux.

Figure 4. Photon flux determined from $\mathrm{Cu}$ and $\mathrm{Ni}$ activities compared with dose measurements and ITS calculation. The activation results sre plotted at the average energy determined by weighting the cross section with the exponential spectral shape of the flux The horizontal bars indicate the giant resonance width which contributes to the activation.

Figure 5. Comparison of LaNSA measured spectra from a thin buried layer target with calculations assuming electroexcitation and exponentially falling electron spectra. 
Fig. 1. T.W. Phillips, Review of Sclentific Instruments 


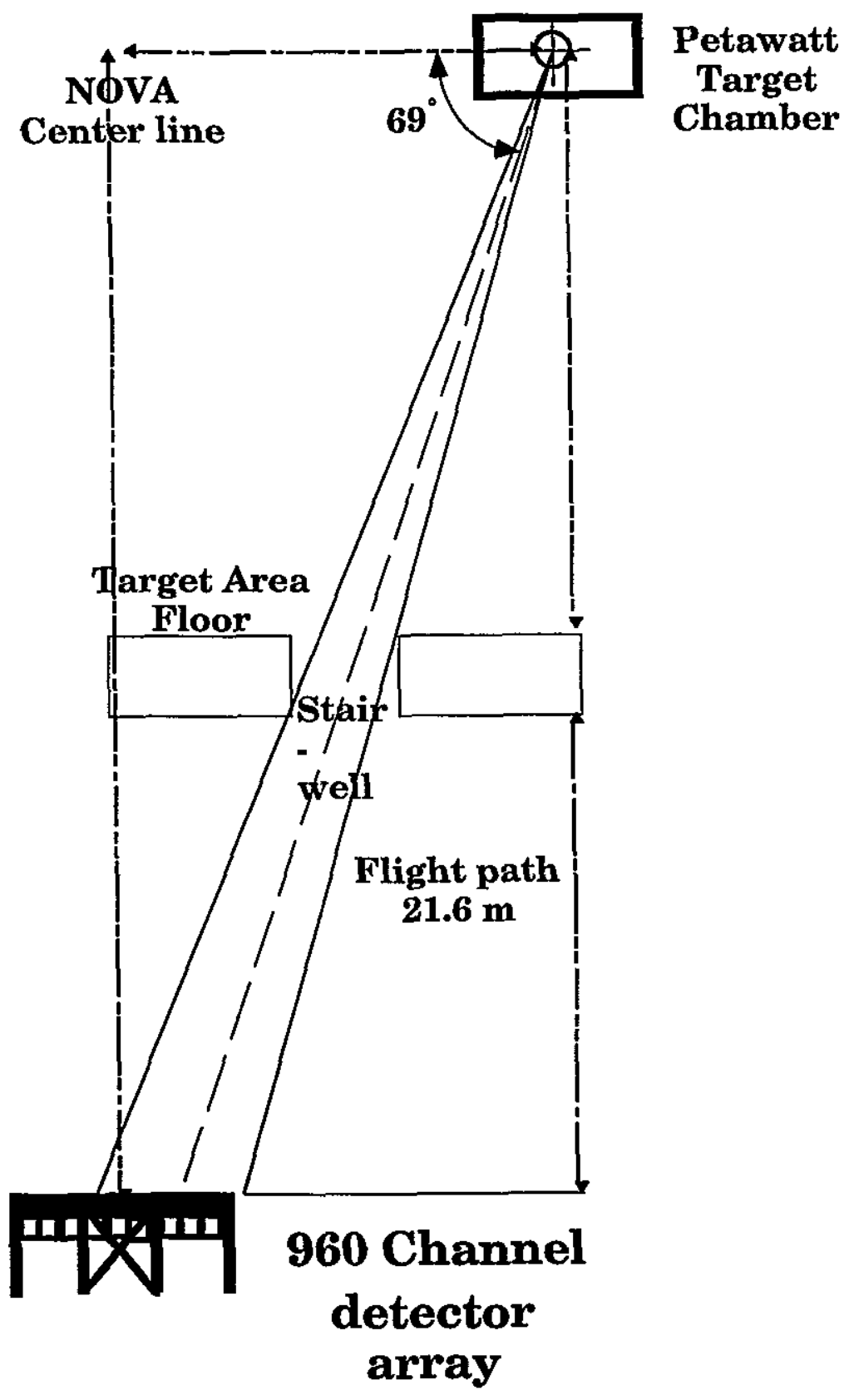


Fig. 2. T.W. Phillips, Review of Scientific Instruments 


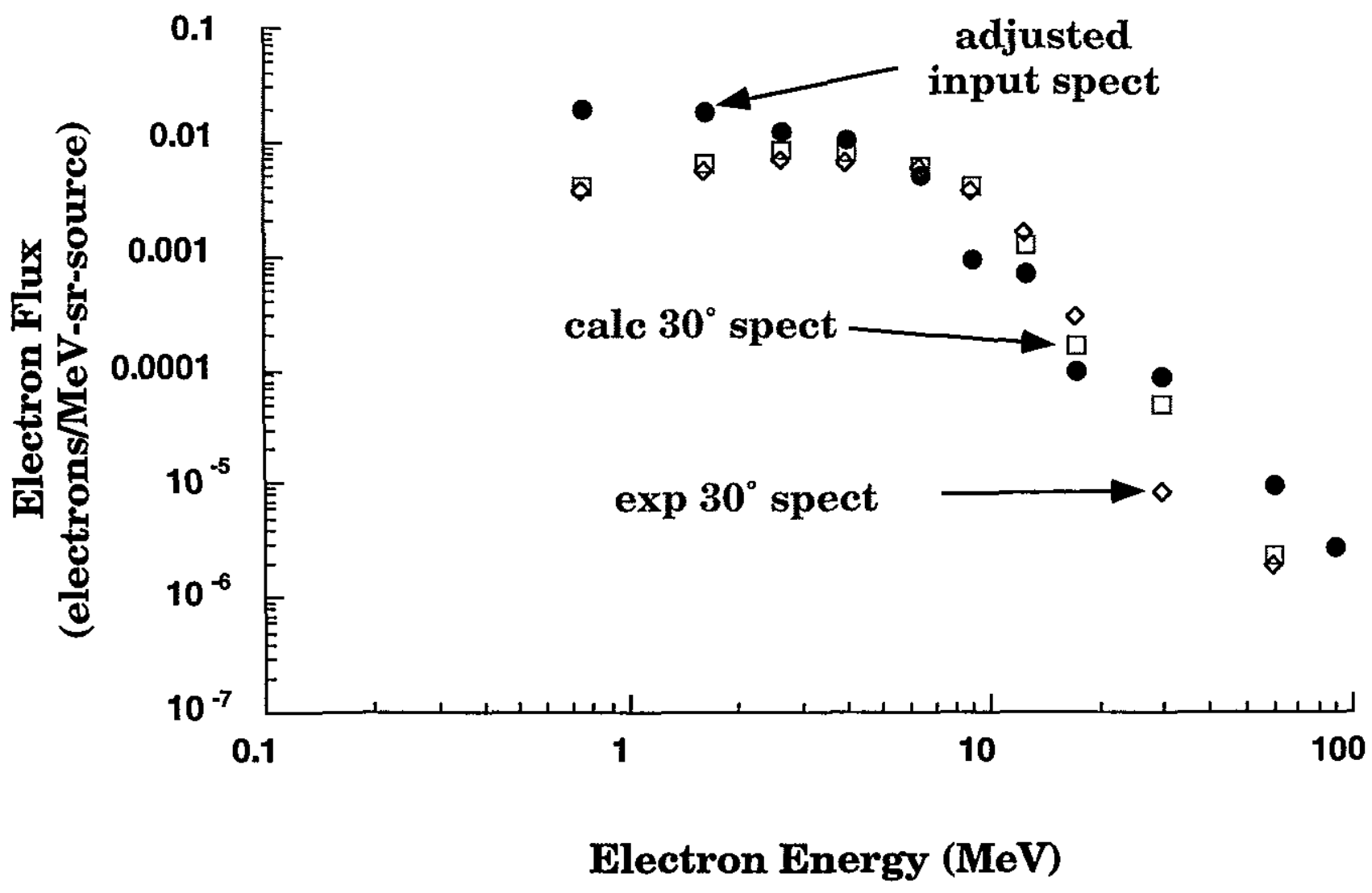


Fig. 3. T.W. Phillips, Review of Scientific Instruments 


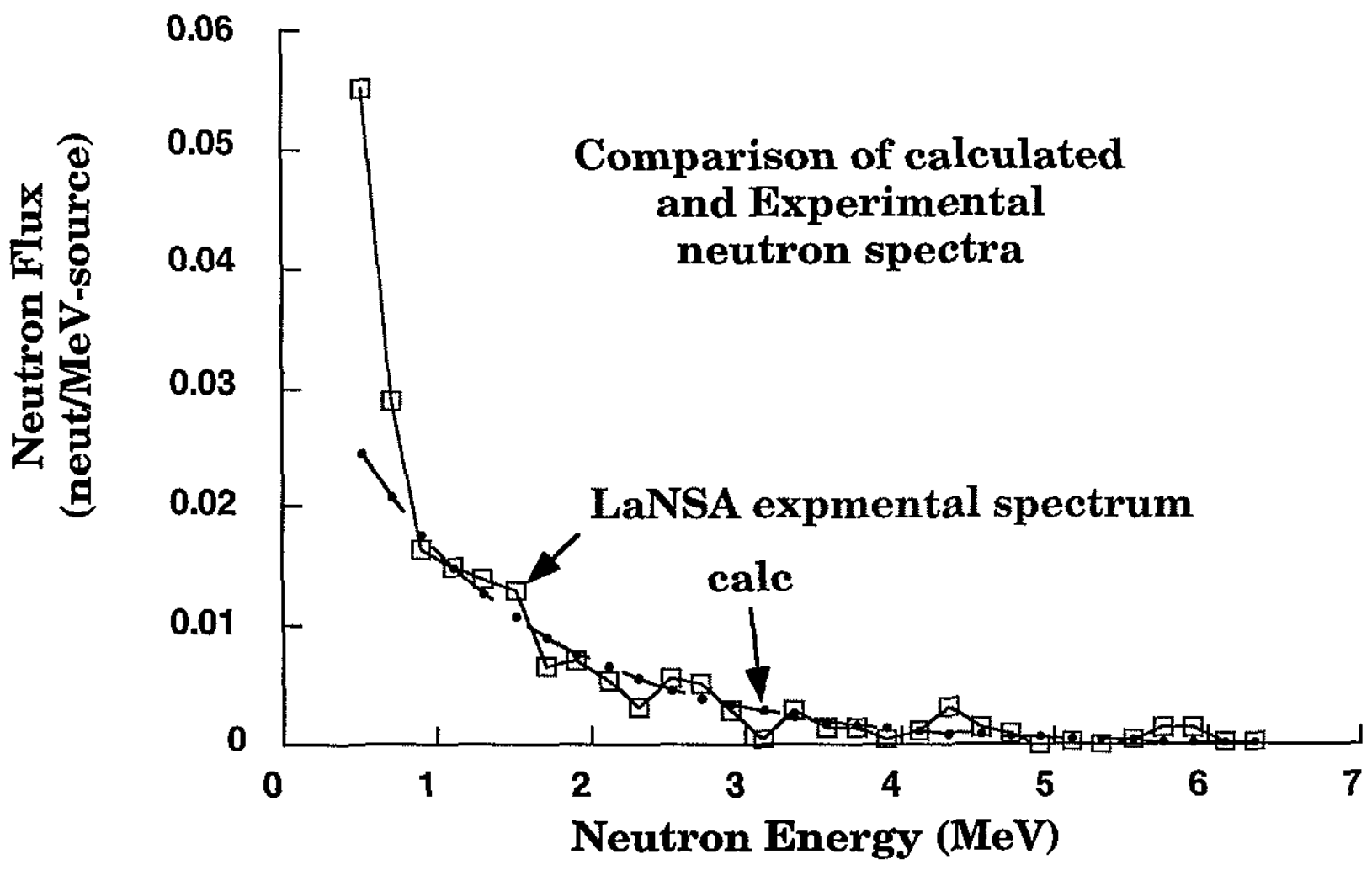

LaNSA yield $\left(E_{n}>1 \mathrm{MeV}\right)=3.45 \times 10^{6}$ Calculated yield $\left(E_{n}>1\right)=3.44 \times 10^{6}$ 
Fig. 4. T.W. Phillips, Review of Scientific Instruments 


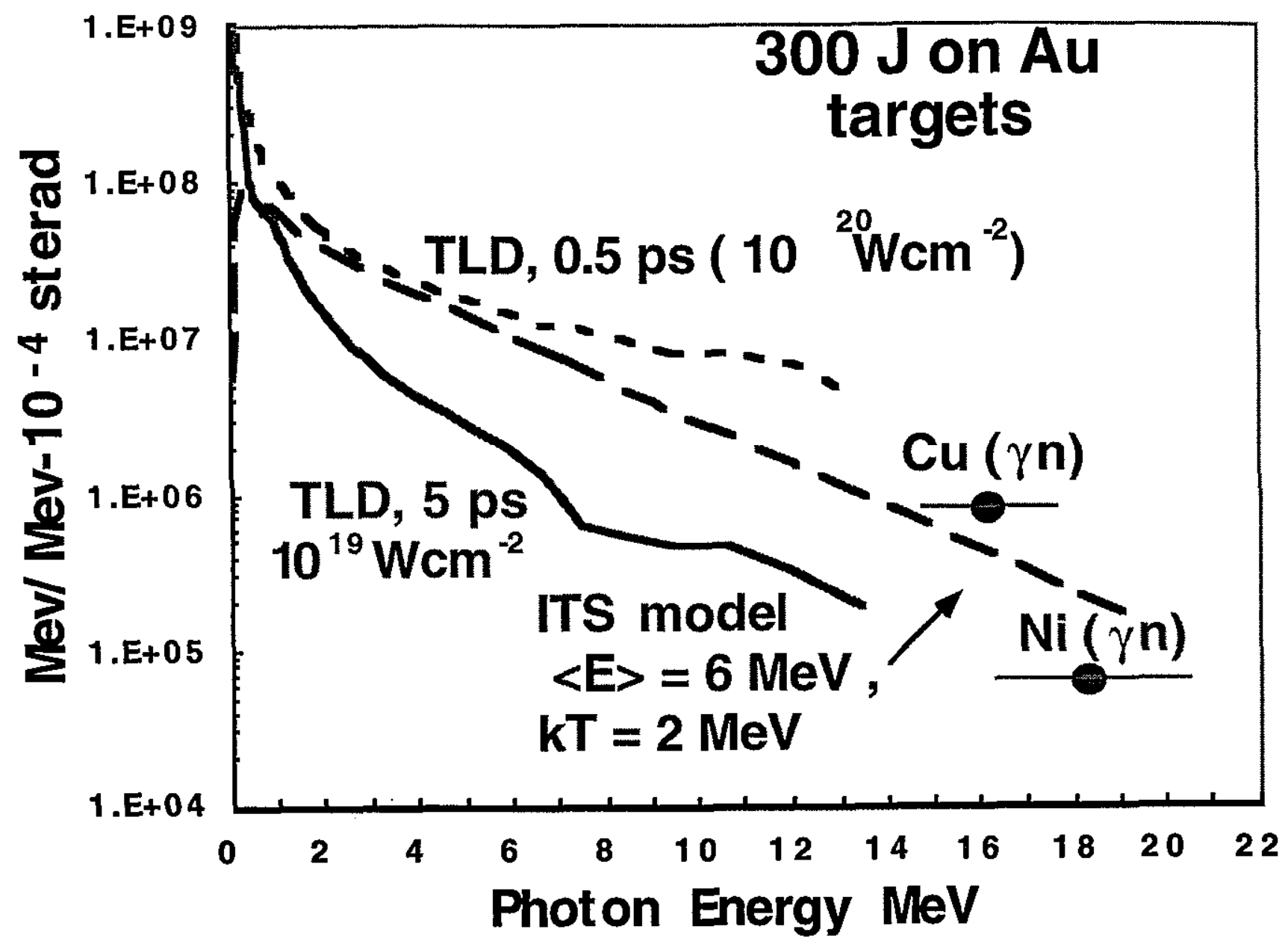


Fig. 5. T.W. Phillips, Review of Scientific Instruments 


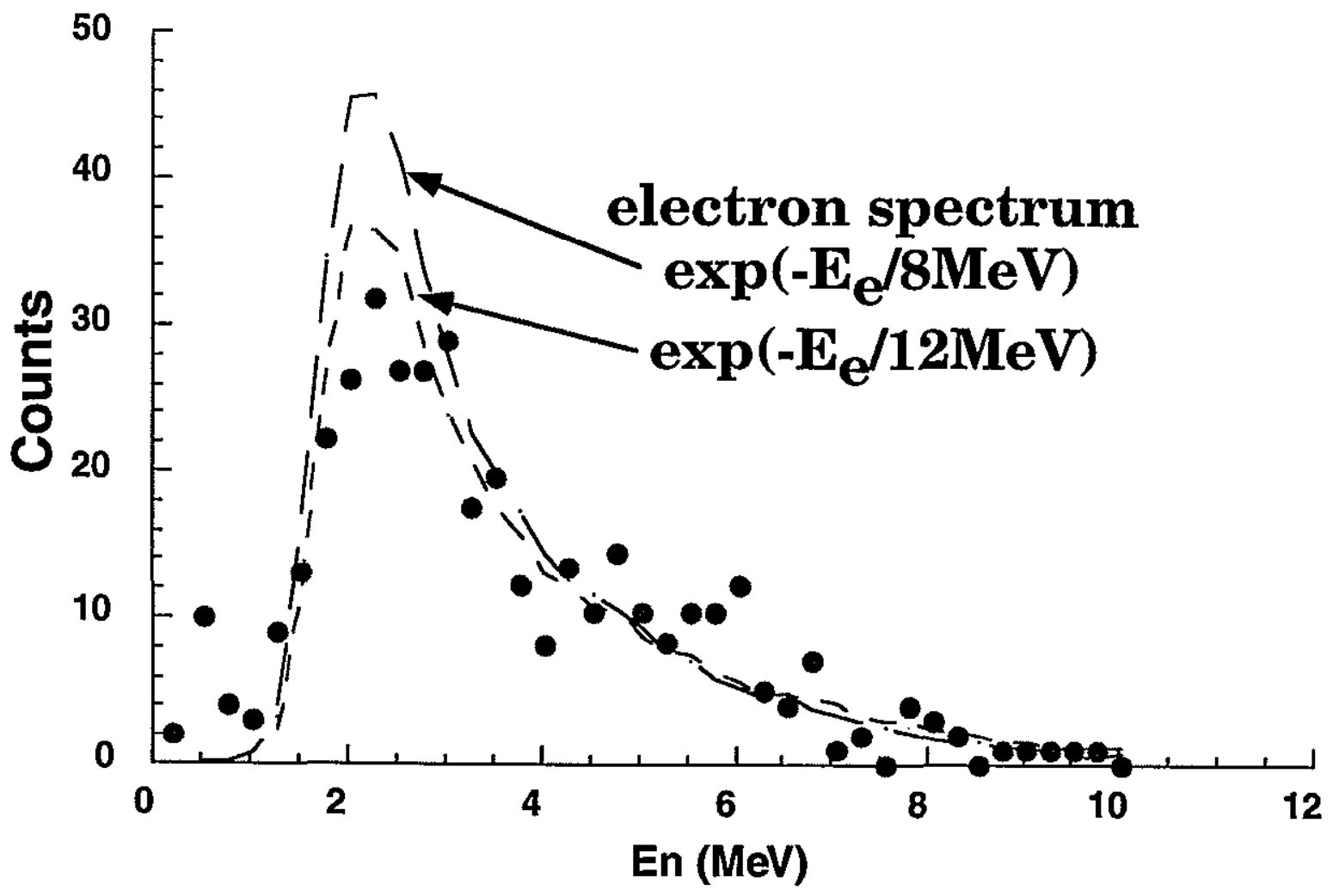




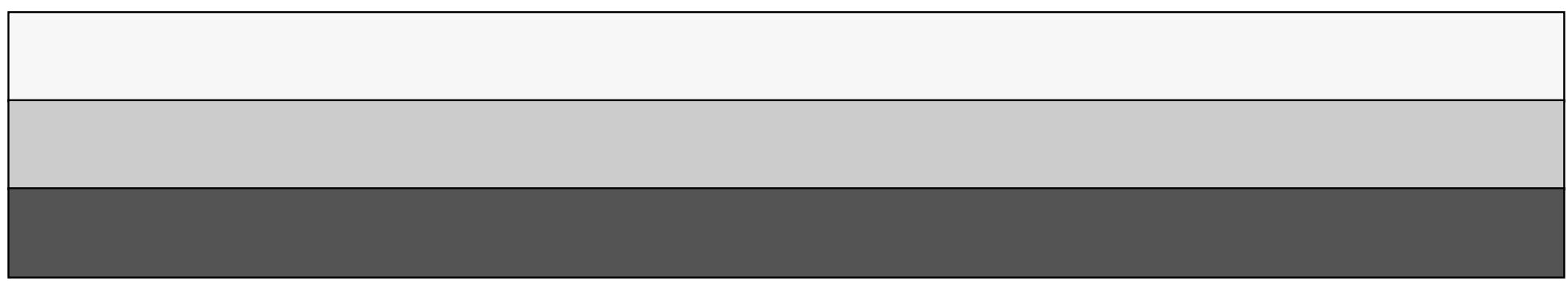

